\title{
Relato de experiência e perfil das condições de saúde e fatores de risco da população masculina participante do projeto saúde do homem - Laranjeiras do Sul / PR
} Experience report and profile of the health conditions and factors of male
population health risk man project participant - Laranieiras do Sul/ PR

Keullin Cristian Oliboni, ${ }^{1}$ Fabiano Popia, ${ }^{1}$ Marcieli Pereira ${ }^{1}$ Universidade Federal da Fronteira Sul, Laranjeiras do Sul, PR, Brasil.

Recebido em: 21/08/2016 / Aceito em: 29/09/2016 / Publicado em: 18/10/2016 keullin.cris@hotmail.com

\section{RESUMO}

Objetivo: descrever o perfil das condições de saúde e fatores de risco dos participantes do projeto saúde do homem, do município de Laranjeiras do Sul/ PR. Método: pesquisa descritivo-exploratória, de abordagem quantitativa, junto a 430 homens atendidos em um programa de saúde do homem. Esses dados foram obtidos em relatórios quadrimestrais e através do Relatório Anual de Gestão. Os dados foram levantados de um total de 794 participantes no projeto, durante o período de setembro de 2013 a setembro de 2014. Resultados: aproximadamente, $28 \%$ dos homens tinham mais de 60 anos; hipertensão arterial (44,9\%); doença mental $(15,6 \%)$; diabetes $(10,5 \%)$ e doença cardiovascular (8,6\%). Considerações finais: verifica-se com este estudo, a necessidade de implementação de ações voltadas à promoção da saúde e ações mais consistentes e acessíveis para a efetivação de uma Política de Saúde do homem.

Palavras-chave: Política; Saúde; Homem.

\section{ABSTRACT}

Objective: to describe the profile of health and risk factors of the participants of man's health project, the city of South Orange / PR. Method: a descriptive and exploratory research with a quantitative approach, along with 430 men met in a men's health program. These data were obtained from quarterly reports and through the Annual Management Report. The data were collected from a total of 794 participants in the project during the period September 2013 to September 2014. Results: approximately $28 \%$ of men over 60 years; hypertension (44.9\%); mental illness (15.6\%); diabetes (10.5\%) and cardiovascular disease (8.6\%). Closing remarks: it appears with this study, the need to implement actions aimed at health promotion and actions more consistent and accessible for the realization of a man's Health Policy.

Keywords: Policy; Health; Man.

\section{INTRODUC̄̃̃O}

A Política Nacional de Atenção Integral a Saúde do Homem (PNAISH) foi instituída por meio da Portaria $\mathrm{GM} / \mathrm{MS} \mathrm{n}^{\circ}$ 1944, de 27 de agosto de 2009 e está voltada prioritariamente para a população masculina na faixa 20 a 59 anos que corresponde atualmente a 52 milhões de brasileiros. A PNAISH propõe qualificar a atenção à saúde da população masculina na perspectiva de linhas de cuidado que resguardem a integralidade da atenção, ou seja, promover, prevenir e assistir os homens compreendendo as suas particularidades. ${ }^{1}$

A PNAISH também reconhece que a população masculina acessa o sistema de saúde por meio de atenção especializada, o que mostra a necessidade de fortalecimento e qualificação da atenção primária, pois os homens não buscam, como fazem as mulheres, os serviços de atenção básica, adentrando os sistemas de saúde pela atenção ambulatorial e hospitalar de média e alta complexidade. Isso resulta em agravo da morbidade pelo retardo na procura e maior custo para os sistemas de saúde. ${ }^{2}$ 
Verifica-se que a doença é considerada como sinal de fragilidade, onde os homens não reconhecem como inerentes à sua própria condição biológica, por isso o homem julga-se invulnerável, sendo exposto a condições de risco. À medida que há aproximação de características de sensibilidade, dependência e cuidado, os homens acreditam que estão predispostos a doenças, lesões e morte. Além disso, os homens sofrem mais de condições graves e crônicas de saúde do que as mulheres. Em consequência disso, vivem menos do que elas. "Há uma relação entre a construção da masculinidade e o comprometimento da saúde dos homens", tal construção e suas implicações na saúde devem ser interpretadas a partir da ótica relacional de gênero. A agressividade relaciona-se a masculinidade erroneamente, porém é concebida de forma natural pela sociedade, podendo assim, transformar-se em "um fator de risco" e "devido à centralidade que o trabalho ocupa na identidade do homem como ser provedor, problemas relacionados ao desemprego podem também comprometer o bem-estar masculino e contribuir para o aumento de suicídios de jovens". ${ }^{3}$

Com o objetivo de implementar a PNAISH, no Município de Laranjeiras do Sul, Paraná, a Secretaria Municipal de Saúde iniciou um conjunto de ações de promoção da saúde direcionadas à população masculina. As atividades preliminares surgiram em agosto de 2013 através da "Campanha Agosto Azul", preconizada pelo Ministério da Saúde. Neste ano, foram abordados os diversos assuntos sobre promoção de saúde e prevenção de doenças, além de oferecer os serviços prestados pela Secretaria Municipal de Saúde. Para realização desta mobilização, formou-se uma equipe multidisciplinar denominada "Patrulha Azul", composta por profissionais do sexo masculino, atuantes na Secretaria de Saúde. A ideia inicial consistia na campanha que fosse realizada de "homem para homem", possibilitando uma melhor aproximação com este público.

Posteriormente, através de discussão e avaliação das ações realizadas, verificou-se a necessidade de um atendimento diferenciado, pois os homens procuravam poucas vezes, as Unidades de Saúde, afirmando que se sentiam envergonhados e ainda que a maioria das ações oferecidas pelos serviços de saúde são voltadas, especialmente, para outros públicos. Além de necessitarem de um horário diferenciado, pois no período de funcionamento das unidades de saúde (das 07:30 hs às 17:00h), quando este público encontra-se cumprindo sua jornada de trabalho.

Em setembro de 2013, foi colocado em prática o Projeto Saúde do Homem, atuando na lógica de facilitar o acesso da população masculina. Por variados motivos de gestão de materiais e de profissionais de saúde, foi definido que a Unidade de Saúde mais centralizada abriria no último sábado de cada mês, e ofereceria aos homens do município atendimento multiprofissional através da equipe da "Patrulha Azul". Essa estratégia obteve êxito e a população aderiu a proposta. Esse movimento teve sequência, durante os meses de outubro e novembro de tal ano, oferecendo atendimento multiprofissional para mais de 100 homens em cada encontro.

No ano seguinte, verificou-se a necessidade de descentralizar esses atendimentos para todas as Uni- dades de Saúde, seguindo a lógica da territorialização e os princípios da Estratégia de Saúde da Família (ESF), a qual está presente em $100 \%$ do território deste município. Buscou-se assim, não somente quantidade, mas sim, maior qualidade nos atendimentos. A partir daí, organizou-se um cronograma, abrangendo todas as unidades, ofertando atendimento por profissionais das ESFs e do Núcleo de Apoio a Saúde da Família (NASF), trabalhando na lógica do vínculo entre a equipe e o usuário e enfatizando o retorno deste paciente para dar sequência às orientações de saúde e tratamento quando necessário.

Após um ano da consolidação do Projeto, é notória a sua importância para o município, visto que a população se encontra extremamente satisfeita com a iniciativa. Porém, verifica-se a necessidade de avaliá-lo, pois até o momento não se tem dados concretos relacionados ao perfil da população masculina atendida pelo Projeto e nem uma linha de base para posteriores comparações das condições de saúde. Dessa forma, o presente trabalho tem o objetivo de descrever o perfil das condições de saúde e fatores de risco dos participantes do projeto saúde do homem, do município de Laranjeiras do Sul/PR.

\section{MÉTODO}

Trata-se de um relato de experiência e uma pesquisa descritivo-exploratória de caráter quantitativo. Este estudo foi realizado no município de Laranjeiras do Sul, localizada no centro do Estado do Paraná. O Município conta com a cobertura de $100 \%$ de Estratégia de Saúde da Família. As 11 equipes de saúde são distribuídas em dois centros de saúde, quatro unidades básicas na cidade e três unidades no interior do município, além de um Núcleo de Apoio a Saúde da Família (NASF), um espaço físico destinado para as ações de Vigilância em Saúde e um Centro de Atenção Psicossocial.

Os dados utilizados nessa pesquisa foram retirados dos relatórios quadrimestrais e do Relatório Anual de Gestão, que foi apresentado pela secretaria Municipal de Saúde e foi analisado e aprovado pelo Conselho Municipal de Saúde. Dessa forma, os dados foram apresentados de forma agregada e sem identificação dos sujeitos. Por terem sido utilizados dados secundários, não houve necessidade de apresentação ao Comitê de Ética.

$\mathrm{O}$ presente estudo utilizou os dados referente aos meses de setembro, outubro e novembro de 2013 e março, maio, julho, agosto e setembro de 2014 .

\section{RESULTADOS}

Para o presente estudo, foi avaliado um ano de operacionalização do projeto de saúde do Homem. Durante esse período, foram realizadas sete ações no Município, com um total de 794 homens atendidos. Destes, foram levantados dados referentes aos fatores de risco à saúde de 430 homens, participantes do Projeto, os quais constam na tabela 1 .

A tabela 1 mostra as condições clínicas existentes, segundo o auto relato dos homens atendidos. Do total de 794 homens atendidos, apenas obtivemos dados de 
Tabela 1 - Condições de saúde e fatores de risco, segundo auto relato, dos homens participantes do "Projeto Saúde do Homem" no Município de Laranjeiras do Sul, PR, 2013-2014.

\begin{tabular}{lc}
\hline Condição de Saúde Existente/fatores de risco & n (\%) \\
\hline Hipertensão Arterial & $193(44,9)$ \\
Doença Mental & $67(15,6)$ \\
Diabetes & $45(10,5)$ \\
Doença Cardiovascular & $37(8,6)$ \\
Doenças Gastrointestinais & $26(6)$ \\
Doença de Próstata & $21(4,9)$ \\
Doenças Pulmonares & $13(3)$ \\
Outras condições Clínicas & $28(6,5)$ \\
\hline
\end{tabular}

430 homens. Os fatores de risco mais proeminentes são hipertensão arterial (44,9\%); doença mental (15,6\%); diabetes $(10,5 \%)$ e doença cardiovascular $(8,6 \%)$.

Cabe ressaltar que a Secretaria Municipal de Saúde de Laranjeiras do Sul, custeou a realização de 470 exames de Antígeno Prostático Específico (PSA), para diagnóstico de câncer de próstata, no período de setembro de 2013 a setembro de 2014. Os critérios utilizados para solicitação do exame foram idade maior de 50 anos; que não tenham realizado o exame nos últimos seis meses; histórico familiar e queixas de sintomas urinários. Destes 470 exames 36 (7,87\%) encontravam-se com o Marcador Tumoral acima dos limítrofes ideais, caracterizando-se assim com alguma alteração na próstata.

\section{DISCUSSÃo}

A realidade da maioria dos municípios brasileiros, incluindo o município de Laranjeiras do Sul, realizam ações que não conseguem atingir os homens; não são exclusivamente destinadas a eles, e ainda assim, os poucos que utilizam estes serviços primários, são idosos, captados, principalmente, pelos programas de acompanhamento a hipertensos e diabéticos. Isto demonstra que a parcela considerada produtiva, principal foco da PNAISH, sempre esteve fora do alcance das ações e serviços, sobretudo aqueles de prevenção e promoção da saúde, tornando crônicos alguns agravos evitáveis.

Para Gomes, Nascimento e Araújo, ${ }^{4}$ os serviços de saúde podem ser considerados pouco aptos em absorver a demanda apresentada pelos homens, pois sua organização não estimula o acesso e as próprias campanhas de saúde não se voltam para este segmento; além disso, o mercado de trabalho, geralmente, não garante formalmente à adoção de tal prática.

Ao observarmos os dados sobre a faixa etária dos homens atendidos, vemos que um dos objetivos do Projeto não vem sendo alcançado, pois a maioria dos beneficiados pelo atendimento e que aderiram ao projeto apresentam uma idade mais avançada e não é a população economicamente ativa, ou seja, que não conseguem se deslocar até a Unidade de Saúde em horário comercial. Sendo assim, é necessária uma reestruturação da ações para um melhor acompanhamento da população masculina no ambiente de trabalho.

Com relação aos dados de Hipertensão arterial, aproximadamente $44,9 \%$ dos homens atendidos apre- sentavam hipertensão arterial, considerado um dos fatores de risco para Doenças Cardiovasculares. Sugere-se, a partir deste dado, uma ampliação das ações de promoção da alimentação saudável e prática de atividade física, os quais podem prevenir ou em caso de já hipertenso, manter a estabilidade da pressão arterial.

Aproximadamente $66 \%$ dos homens participantes do projeto apresentavam sobrepeso e obesidade e que é superior aos dados encontrados pela Pesquisa de Vigilância de Fatores de Risco e Proteção para Doenças Crônicas por Inquérito Telefônico ${ }^{5}$ mostra que o percentual de pessoas com excesso de peso superou, pela primeira vez, mais da metade da população brasileira, identificando que $51 \%$ da população acima de 18 anos está acima do peso ideal, sendo que entre os homens, o excesso de peso atinge $54 \%$.

A prevalência de tabagismo relatada $(29,4 \%)$ foi superior ao percentual atingido pela capital do estado que tem o segundo maior índice de fumantes entre as capitais, $20 \%$. A pior taxa fica com Porto Alegre, onde $23 \%$ da população é dependente do cigarro. A partir desta análise, indica-se o fortalecimento dos Grupos Anti-tabaco já existentes no município, além da capacitação de novos profissionais, ampliando assim o acesso ao tratamento e acompanhamento para os homens que desejam deixar de fumar.

A ingestão de bebidas alcoólicas também foi superior $(31,6 \%)$ aos dados fornecidos pelo levantamento da Vigetel, em que aponta que aumentou o consumo excessivo de bebidas alcoólicas no país, passando de $16,2 \%$ para $18 \%$ da população, entre 2006 e 2010. Entre os homens, a proporção passou de $25,5 \%$ para $26,8 \%$. Tanto o hábito de fumar, quanto o exagero na bebida são indicadores importantes no monitoramento dos fatores de risco para doenças crônicas não transmissíveis, como hipertensão arterial, diabetes, problemas cardíacos e acidente vascular cerebral hemorrágico. ${ }^{5}$

O sedentarismo esteve presente em $60 \%$ dos homens, que participaram do projeto e também foi superior aos dados do Vigitel que mostram que cerca de $50 \%$ dos homens são sedentários. O acompanhamento dos homens que apresentaram resultados alterados do exame PSA não pode ser analisado, tendo em vista que as informações individuais não foram analisadas nesse artigo.

Após identificar o perfil da população masculina participante do Projeto, se faz necessário observar os indicadores de mortalidade entre homens. Em Laranjeiras do Sul, segundo dados do Sistema de Informações sobre Mortalidade - SIM, no ano de 2013 ocorreram 244 óbitos, sendo que 140 eram homens (57,38\%). Destes, as seis principais causas de óbito informadas foram, em primeiro lugar com 20,71\% causadas por Neoplasias, em segundo, com $20 \%$, as Doenças do aparelho respiratório, em terceiro com 19,28\% Doenças do aparelho circulatório, em quarto com $14,28 \%$ por Causas Externas, em quinto com $5 \%$ por Doenças do aparelho digestivo, em sexto e não menos importante, com 3,57\% por Transtornos mentais e comportamentais.

A partir desta análise, pode-se fazer um comparativo entre os principais agravos que acometem a saúde dos homens atendidos pelo projeto e as principais causas de óbito. Verificando uma homogeneidade nas informações, em que os fatores de risco como tabagismo, alcoolismo, sedentarismo e IMC elevado, influenciam 
no aparecimento das doenças crônicas do aparelho circulatório e respiratório as quais somam juntas, quase $40 \%$ das causas óbito.

\section{CONSIDERACִÕES FINAIS}

O Projeto Saúde do Homem do município de Laranjeiras do Sul vem contribuindo para mudança deste cenário, oferecendo atendimentos de equipe multiprofissional e facilitando o acesso na Atenção Básica, buscando assim, diminuir a procura pela média e alta complexidade.

A partir dessa análise preliminar da saúde do homem no Município, sugere-se a ampliação de ações de promoção da saúde; reestruturação das ações do setor de Vigilância em Saúde do Trabalhador, adequando-as e oferecendo condições para um melhor acompanhamento da população masculina no ambiente de trabaIho; ampliação das ações de promoção da alimentação saudável e prática de atividade física; fortalecimento dos Grupos Anti-tabaco já existentes no município, além da capacitação de novos profissionais, ampliando assim o acesso ao tratamento e acompanhamento para os homens que desejam deixar de fumar; efetivação das ações de Educação em Saúde para população, além de maior esclarecimento sobre os malefícios do álcool; incentivo para um melhor acompanhamento dos pacientes que apresentam exame PSA alterado, pela equipe da Estratégia Saúde da Família, buscando manter o vínculo, mesmo que o acompanhamento seja direcionado para média e alta complexidade em outros municípios.

Uma das principais limitações desse deste estudo foi o elevado percentual de informações incompletas, as quais influenciaram significativamente e limitaram a realização de uma análise mais complexa. Dessa forma, para otimização dos dados coletados propõe-se a implantação de um sistema de informação que irá armazenar e realizar o cruzamento dos mesmos, gerando relatórios e obtendo novos parâmetros para posteriores ações.

Sendo assim, o Projeto Saúde do Homem visa mudar essa situação, incentivando uma maior aproximação das equipes de saúde para com os homens de diferentes faixas etárias, buscando oferecer um olhar diferenciado a esta população, ampliando o acesso, oferecendo atendimentos em horário diferenciado e buscando facilitar a adesão destes usuários.

\section{REFERÊNCIAS}

1. Brasil. Ministério da Saúde. Secretaria de Atenção à Saúde, Departamento de Ações Programáticas Estratégicas - Política Nacional de Atenção Integral à Saúde do Homem: Princípios e Diretrizes, Brasília, 2008.

2. Carrara S, Russo J, Faro L. A política de atenção à saúde do homem no Brasil: os paradoxos da medicalização do corpo masculino. Physis 2009;19(3):659-78. doi: 10.1590/S010373312009000300006.

3. Gomes R, Nascimento EF. A produção do conhecimento da saúde pública sobre a relação homem-saúde: uma revisão bibliográfica. Cad. Saúde Pública 2006;22(5):901-11.

4. Gomes R, Nascimento EF, Araujo FC. Por que os homens buscam menos os serviços de saúde do que as mulheres? As explicações de homens com baixa escolaridade e homens com ensino superior. Cad. Saúde Pública 2007;23(3):565-74.

5. Brasil. Ministério da Saúde. Secretaria de Vigilância em Saúde. Vigitel Brasil 2011: Vigilância de Fatores de Risco e Proteção para Doenças Crônicas por Inquérito Telefônico. Ministério da Saúde, Secretaria de Vigilância em Saúde Brasília: Ministério da Saúde, 2012.

Como citar: OLIBONI, Keullin Cristian; PAPIA, Fabiano; PEREIRA, Marcieli. Perfil da população masculina participante do projeto Saúde do homem - Laranjeiras do Sul / PR. Cinergis, Santa Cruz do Sul, v. 18, n. 1, out. 2016. ISSN 2177-4005. Disponível em: <https://online.unisc.br/seer/index.php/cinergis/article/view/8159>. Acesso em: 11 out. 2016. doi:http:// dx.doi.org/10.17058/cinergis.v18i1.8159. 